\title{
Multiple-Computer User Interfaces: A Cooperative Environment Consisting of Multiple Digital Devices
}

\author{
Jun Rekimoto \\ Sony Computer Science Laboratory Inc. \\ Takanawa Muse Building \\ 3-14-13 Higashigotanda, Shinagawa-ku, Tokyo 141 Japan \\ $+81354484380$ \\ Email: rekimoto@csl.sony.co.jp \\ URL: http://www.csl.sony.co.jp/person/rekimoto.html
}

\begin{abstract}
Traditional graphical user interfaces (GUIs) are mainly designed for an environment consisting of a single display and a set of single input devices. However, in the near future we will be using a number of computers (and other electronic devices). Just as we often combine several physical devices to perform a task in a real world, it should also be possible to use multiple computers more effectively. This paper proposes the notion of multiple-computer user interfaces in a future cooperative environment. We also describe an interaction technique that allows a user to transfer digital information to other electronic devices placed in a physical environment, and a digital whiteboard system that effectively uses a multiple-device architecture.
\end{abstract}

Keywords. multiple-device user interfaces, ubiquitous computing, pick-anddrop, digital whiteboard systems, cooperative buildings

\section{Introduction: From a single-device UI to a multi-device UI}

As Mark Weiser (1991) and many other visionaries foresaw, our physical space will be filled with a number of digital devices. Some of them will be invisible (i.e., embedded in the environment); others will remain visible like today's desktop computers. In a meeting room today, for example, participants often use a notebook PCs while presenters use a digital whiteboard. Even now, in individual work environments, we often use more than one computer at a time (such as a desktop PC and a PDA).

In our daily lives, it is natural to combine several physical "tools" to achieve a task. However, it is not so easy to combine multiple computers and devices. One reason is that computers (and other electric devices) are not designed to be used in multiplecomputer (multiple-device) environments. For example, even simple GUI techniques such as cut-and-paste or drag-and-drop do not work across computer boundaries. 


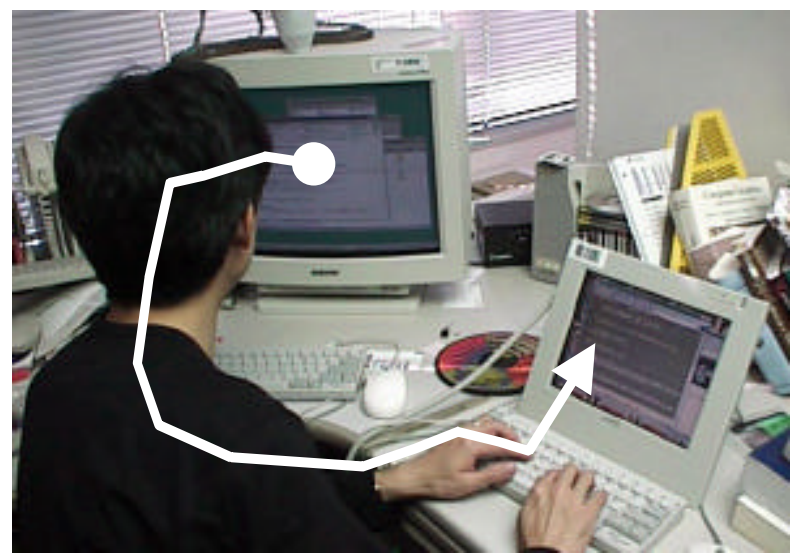

Fig. 1 Data transfer through the human body? A typical difficulty when dealing with two or more computers in a same place.

Fig. 1 shows a typical difficulty one encounters when using more than one computer. The user here reads a text segment such an URL on the left display, and then types it into the computer at the right, even though these two computers are connected to the network. Since there is no easier way (such as drag-and-drop) to transfer data between the two, he prefers to transfer the data by hand. One survey revealed that more than $60 \%$ of the software engineers in Sony's development division experience similar problems.

We believe that future computer interfaces must be designed to be used in a multiple-computer (or multiple-device) environment. More specifically, interaction techniques must overcome the boundaries among multiple devices, and that environments must accommodate to the dynamic addition of new devices.

Another important issue is a physical aspect of computer devices. For example, we can not simply apply the same UI design for small (palmtop sized) and large (wallsized) computers, just as memo-pads and whiteboards are used quite differently in our everyday world. Physical position and direction of computer devices are also important. A display in a public meeting space would be used as a shared digital bulletin board for a group, while a small screen device attached on the wall of a personal work space would show schedule of an individual. When a table-top becomes a computer display, it would be meaningless to add a "menu-bar" on top of the (table) screen, because there is no notion of "upper-side" of the computer display.

In this paper, we describe our recent attempts in designing interaction techniques and systems based-on the notion of multiple-computer user interfaces. In the next section we will discuss a data-transfer technique (called Pick-and-Drop) between multiple computers, followed by a description of a digital whiteboard design using the multiple-device approach. 


\section{Pick-and-Drop: an interaction technique for transferring digital information in a physical environment}

The Pick-and-Drop is our initial attempt to design a multiple-computer user interface (Rekimoto 1997), focusing on data transfer problems in multi-comp environments. Like the commonly used drag-and-drop, pick-and-drop is a pen-based direct manipulation technique for transferring digital data such as icons on a computer screen. Unlike drag-and-drop, pick-and-drop allows a user to physically pick up a digital object from one screen, carry it through real space, and drop it in a different place, or even on a different computer screen. Just like chopsticks are used for moving a piece of food from one dish to another, pick-and-drop provides a method whereby a user can physically carry data as if it were a real object.

Although these operations can also be implemented by using remote copy or shared file systems, we feel that pick-and-drop offers more natural and direct way to manipulate a digital object in a real space. Using traditional data transfer methods, users have to deal with many symbolic concepts such as machine names, file names, and directory path names, even if target computers are physically placed side-by-side. On the other hand, data transfer with pick-and-drop is more direct and visible as opposed to symbolic. Users can be aware of physical position on computer screens, just like moving a physical object in a real world.

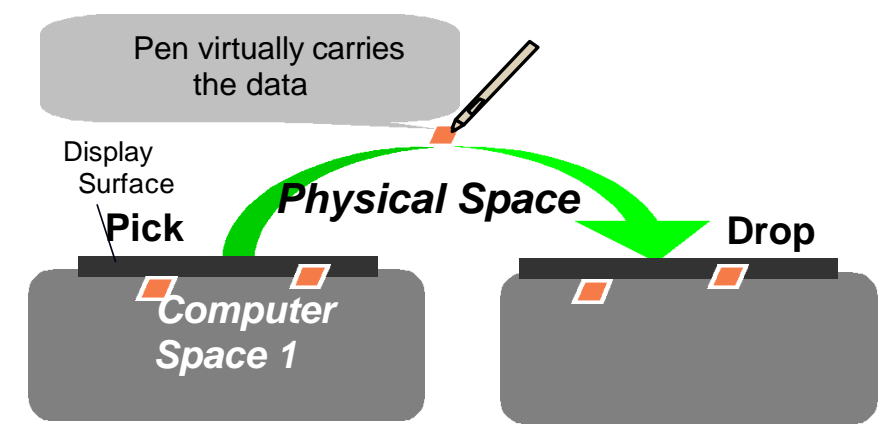

Fig. 2. Pick-and-Drop supports data transfer among multiple devices through the physical space

We developed the multi-computer Pick-and-Drop without adding actual storage capabilities to the pen device, by introducing the concept of Pen IDs. In our design, each pen is assigned a unique ID. We are currently using a combination of modifier buttons (attached to the pen as a side switch) to represent IDs. Based-on the WACOM electromagnetic stylus technology, this pen ID as well as the pen position is readable from the computer (screen) when a pen is closer enough to its screen. We also assume that all computers are connected to the network (either wired or wireless). There is a server called the "pen manager" on the network. 

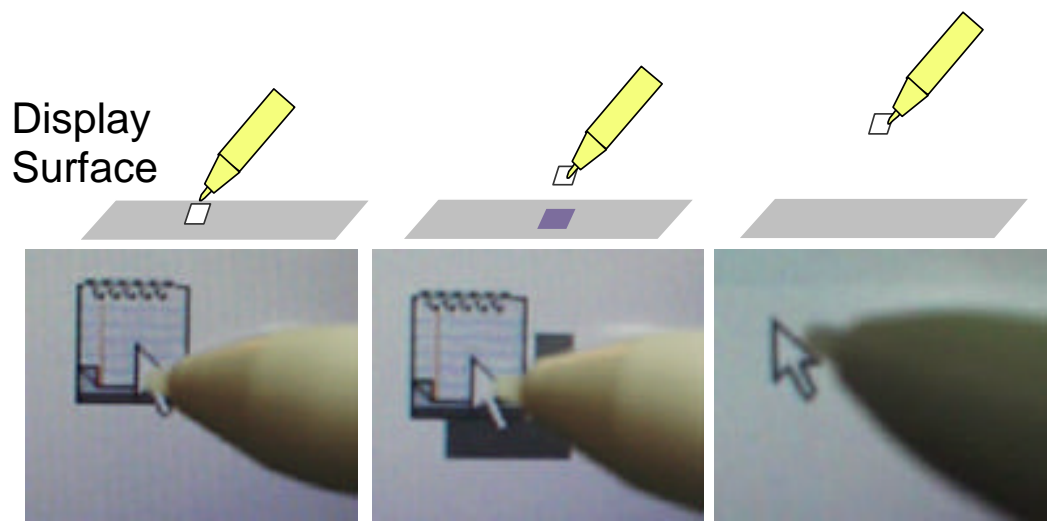

Fig. 3. A data icon with shadow indicates pen holding data without dropping it on the screen. (left: the pen touches the screen, and dragging an icon, middle: the pen is holding data near the screen, and an icon with shadow appears on the screen to show the pen's data type, right: the pen moves away from the screen, and the icon disappears)

When a user taps an object (typically an icon) on the screen with the pen, the pen manager binds its object ID to the pen ID. This binding represents a situation in which the pen virtually holds the object (even though the pen itself does not contain any storage). When the user moves the same pen towards the other display, the pen manager supplies the type of the bound object to the display. Then the data icon with shadow appears on the display below the current pen position to show the data the pen is holding, before actually dropping it on the screen (Fig. 3). At this moment, the pen does not touch the screen. Finally, when the user touches the display with the pen, the pen manager asks the first computer to transfer the data to second computer.

Since each pen has its own ID, simultaneous Pick-and-Drop operations by more than one pen can overlap. This feature would be useful in a collaborative setting.

\section{Applications of Pick-and-Drop}

Once data transfer between multiple devices becomes widely available, we will have greater flexibility in designing a physical work environment by combining multiple digital devices (Fig. 4 shows typical usage of pick-and-drop between different types of computers). For example, a computer augmented drafting desk could consist of a large main display and several small palm-sized computers. These small computers would act as temporary work places for drawing data (i.e., tangible paste buffers), tool palettes, or data-carrying devices to store and transfer drawing materials. The user could physically deploy these small devices around the main display to con- 

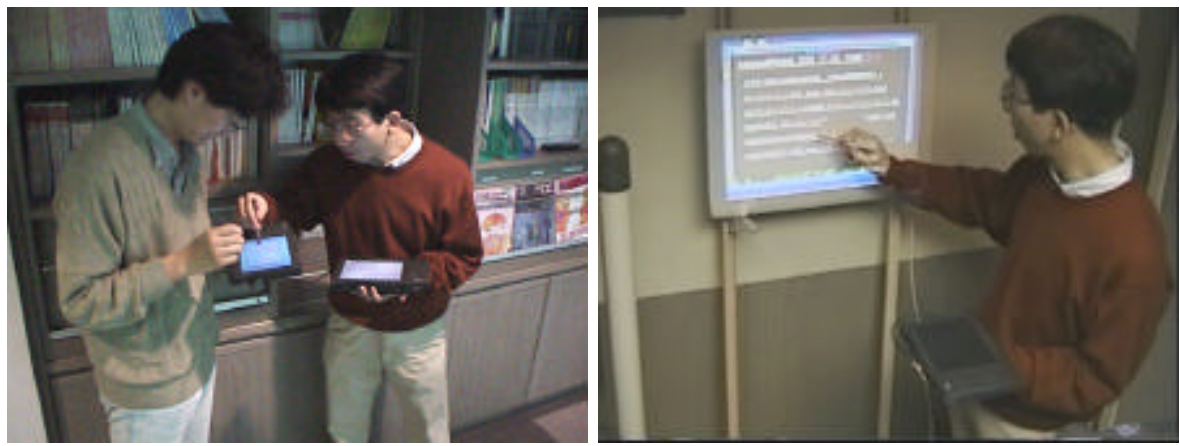

Fig. 4. Applications of Pick-and-Drop (left: transferring data between two PDAs, right: picking up information from the kiosk terminal)
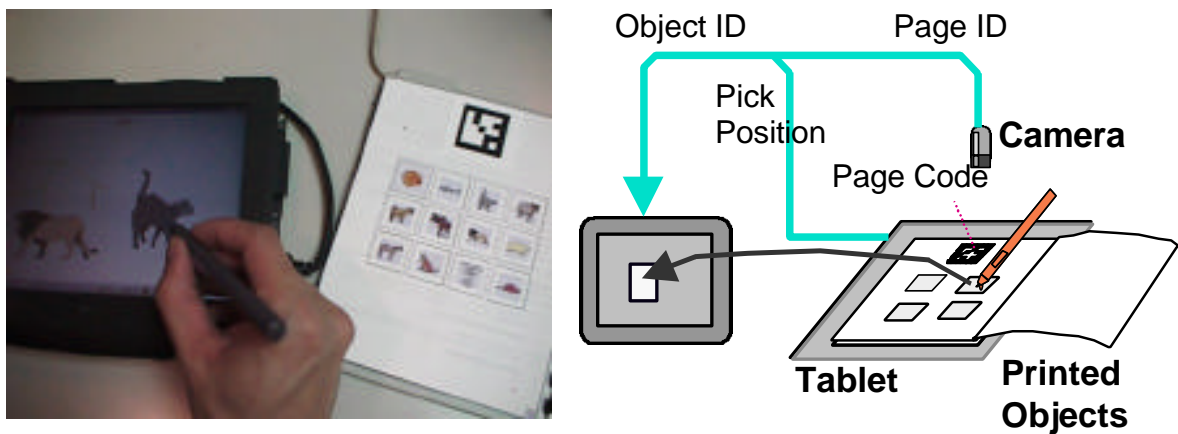

Fig. 5. PaperIcon: Data transfer between printed material and a computer screen.

figure his/her own work environment. Data transfer among all the devices would be supported by the pick-and-drop.

We also have implemented data transfer from paper materials to digital surfaces using a combination of a tablet and a camera (Fig. 5). The printed page is placed on a pen sensitive tablet and a camera is mounted over the tablet. The camera is used to identify the opened page by reading an ID mark printed on it. The user can freely flip through the booklet to find a desirable icon. The system determines which icon is picked based on the page ID and the picked position on the tablet. With this technology, even physical material such as clip-art books can be a part of a multiplecomputer environment. 

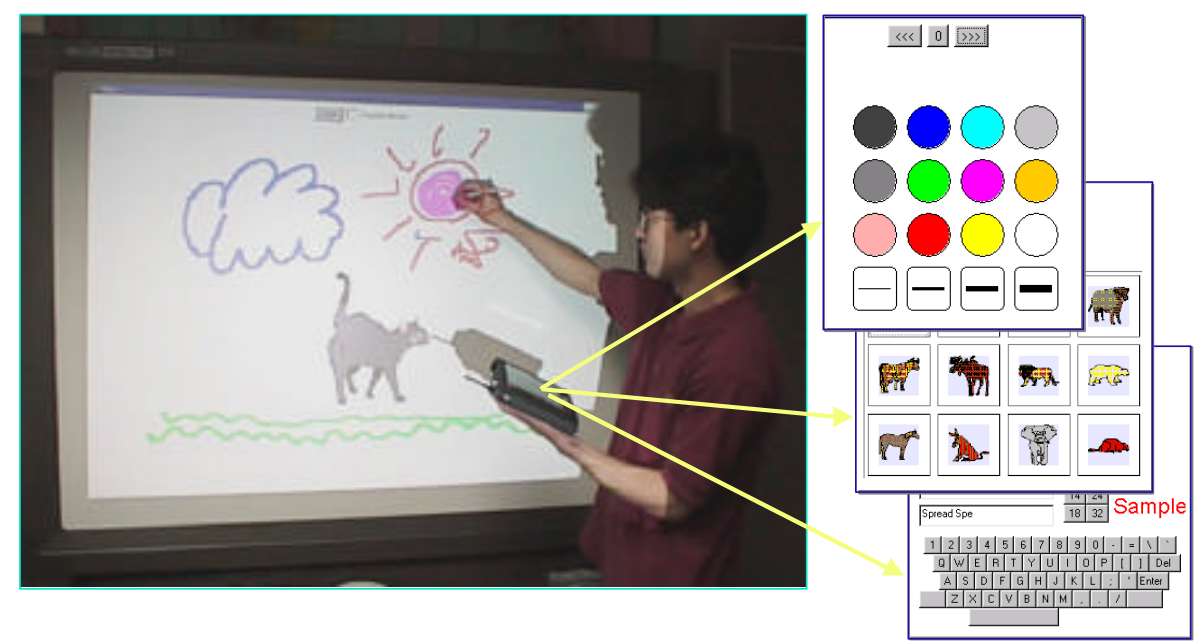

Fig 6. A digital whiteboard system using the multiple-device architecture. Just as an oilpainter effectively uses a palette in his/her hand, the hand-held device offers an easy way to create a new text/graphics object, to select existing data from network, to select pen attributes, and to control the whiteboard application. Note that the same pen can be used to manipulate both of the whiteboard and the hand-held computers.

\section{Applying a multi-computer configuration to a digital whiteboard design}

Another possible multi-device configuration is to use a hand-held tablet as a support for large (whiteboard-sized) display interfaces (Rekimoto 1998).

Traditional digital whiteboard systems such as Xerox LiveBoard (Elrod et al. 1992) often suffer from a limited capability to enter text and the handling of existing data. The large display surface of the whiteboard also makes traditional GUI design ineffective. For example, placement of menu bars or tool bars becomes a problem because they might be out-of-reach from users. Single thread features of current GUI design also prohibits parallel activities among collaborators.

Existing digital-whiteboard systems have tried to address these problems by enhancing the design of user interfaces only on a whiteboard. However, we feel that if some of the whiteboard functions were given to the participants via hand-held devices, it would offer a simple, yet powerful solution to these problems.

Based-on this multiple-device design, we have developed a digital whiteboard system using a palm-sized computer as a control palette (Fig. 6). The user can select a color and brush type for the pen by tapping the control panel on the palm-sized 


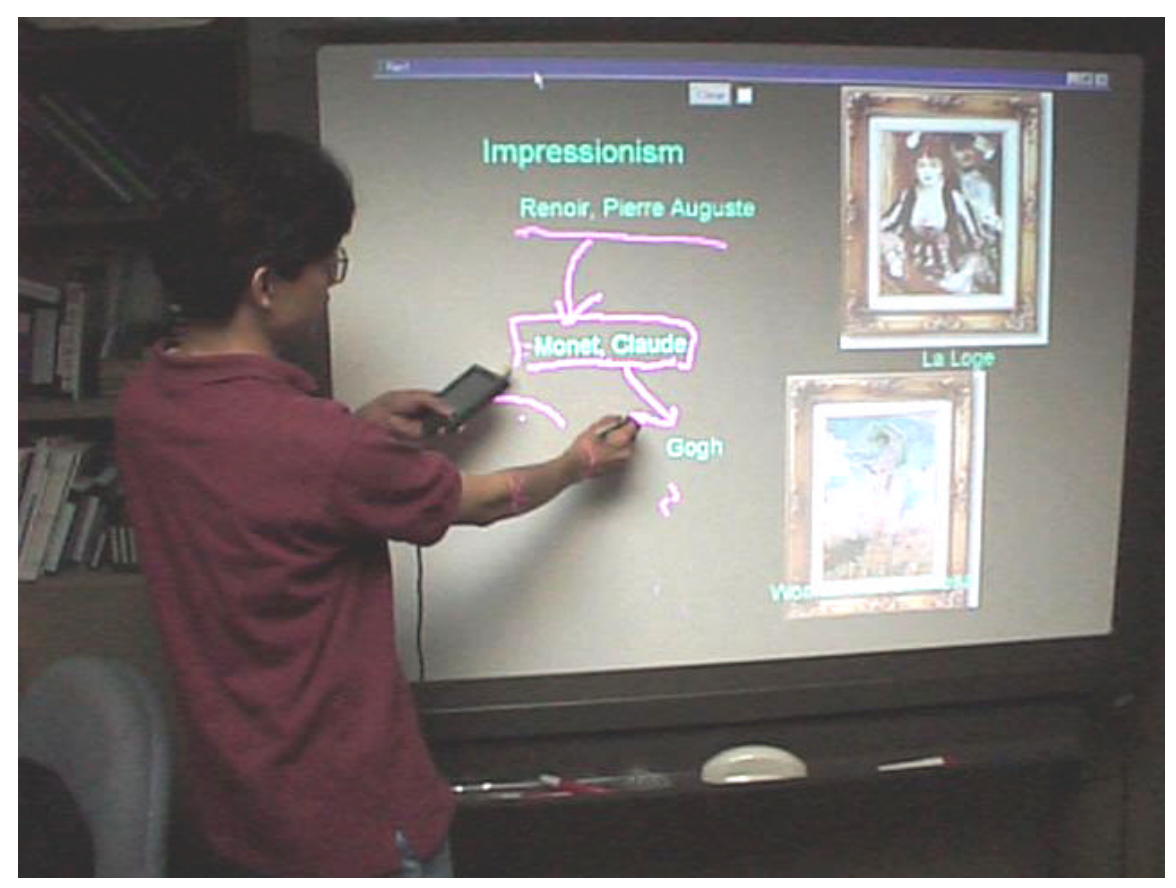

Fig. 7. A lecture-style session using a PDA in the left hand and a digital pen in the right hand. A lecturer can select text or graphics items using a PDA, then paste it on the whiteboard with the left hand. Note that the PDA contains a position sensor and the user can specify the paste position by placing the PDA on the whiteboard.

tablet. This metaphor is similar to physical oil painting using a canvas and a palette. This interaction style is advantageous for drawing on a large display, because users do not have to click on a tool-palette on the whiteboard, which might be out of reach. The user can also transfer digital data between any participating computers (e.g., whiteboard, palmtop, and other user's palmtop), using pick-and-drop operations.

Fig. 7 is another variation of a digital whiteboard that also employs a multipledevice configuration. This setting is designed for a lecturer in a classroom. The system allows a lecturer to select and attach data elements from the screen of the Pilot PDA in the left hand to the whiteboard. A lecturer can freely decide which data should be displayed next, according to the flow of his/her talk. The lecturer can also add hand-drawn diagrams or texts using a pen in his/her right hand. The result becomes a mixture of a whiteboard-based lecture style with digital presentation information. Such an effect can not be realized by using only traditional presentation software. 


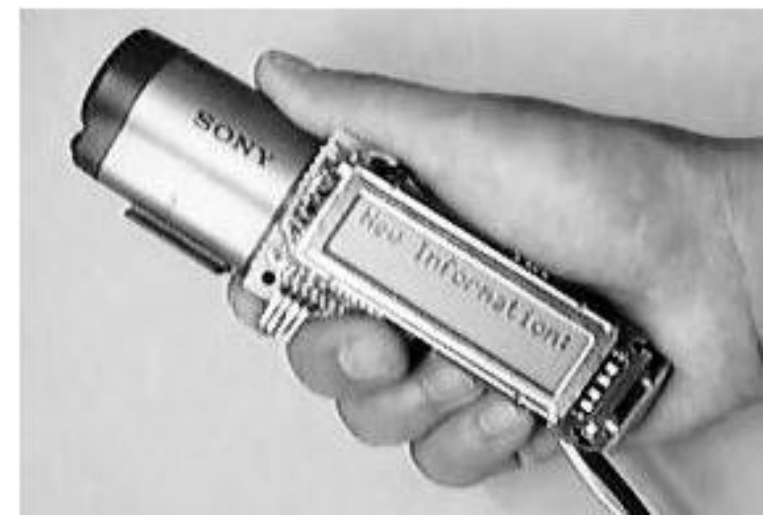

Fig. 8. A prototype data picking wand

\section{Summary and future projects}

In this paper, we have argued the importance of multiple-computer user interfaces in future cooperative environments. We also have described an interaction technique based on a metaphor of digital-physical fusion, called the Pick-and-Drop, with several example applications. We are currently working on extending the concept of information picking to the more general domain.

Fig. 8 Shows the prototype data picking "wand" --- a new style of future PDAs. Using the combination of camera and infrared sensors, it supports data transfer between several digital devices (e.g., VCR and computer) as well as non-digital materials (e.g., a physical bulletin board and a document cabinet) in our daily environment.

\section{References}

1. Mark Weiser: The Computer for the Twenty-first Century. Scientific American, vol. 265, number 3, pp.94-104. (1991)

2. Jun Rekimoto: Pick-and-Drop: A Direct Manipulation Technique for Multiple-computer Environments, Proceedings of UIST'97, pp.31-39 (1997).

3. Jun Rekimoto: A Multiple Computer Approach for Supporting Whiteboard-based Interactions, Proceedings of CHI'98 (in press) (1998).

4. Scott Elrod, Richard Bruce, Rich Gold, David Goldberg, Frank Halasz, William Janssen, David Lee, Kim McCall, Elin Pedersen, Ken Pier, Jhon Tang, and Brent Welch. LiveBoard: A large interactive display supporting group meetings, presentations and remote collaboration, Proceedings of CHI'92, pp. 599-607, (1992). 